\title{
Diagnostic accuracy of liquid-based endometrial cytology in the evaluation of endometrial pathology in postmenopausal women
}

\author{
C. Remondi*, F. Sesti*, E. Bonanno ${ }^{\dagger}$, A. Pietropolli* and E. Piccione* \\ *Section of Gynecology, Academic Department of Biomedicine \& Prevention and Clinical Department of Surgery, Tor Vergata \\ University Hospital, Rome, Italy and ${ }^{\dagger}$ Anatomic Pathology Institute, Department of Biomedicine $\&$ Prevention, School of \\ Medicine, Tor Vergata University Hospital, Rome, Italy
}

Accepted for publication 3 August 2012

C. Remondi, F. Sesti, E. Bonanno, A. Pietropolli and E. Piccione

Diagnostic accuracy of liquid-based endometrial cytology cytology in the evaluation of endometrial pathology in postmenopausal women

Objective: The aim of this study was to compare liquid-based endometrial cytology with hysteroscopy and endometrial biopsy regarding its diagnostic accuracy in a series of postmenopausal women with abnormal uterine bleeding (AUB) or asymptomatic women with thickened endometrium assessed by transvaginal ultrasound as a screening procedure.

Methods: Inclusion criteria were: menopausal status; the presence of AUB and/or thickened endometrium assessed by ultrasound (cut-off $4 \mathrm{~mm}$ ); a normal Papanicolaou (Pap) smear; and no adnexal pathology at ultrasound. Exclusion criteria were: previous endometrial pathology; and previous operative hysteroscopy. Of 768 postmenopausal women referred to our general gynaecology clinics, 121 fulfilled the inclusion criteria and were recruited to the trial. Twenty-one refused to participate. Cytological sampling was carried out by brushing the uterine cavity using the Endoflower device with no cervical dilation and the vial was processed using a ThinPrep ${ }^{\circledR} 2000$ automated slide processor. The slides were stained using a Pap method.

Results: In 98 cases with histological biopsies, endometrial cytology detected five cases of endometrial carcinoma, 10 of atypical hyperplasia and 47 of non-atypical hyperplasia; 36 cases were negative. In two cases cytology was inadequate because of uterine cervical stenosis. Taking atypical hyperplasia or worse as a positive test and outcome, the diagnostic accuracy of the endometrial cytology was $93.5 \%$, with a sensitivity of $92 \%$ and specificity of $95 \%$, a positive predictive value of $73 \%$ and a negative predictive value of $99 \%$. All the carcinomas were detected by cytology. Only $42 \%$ of women with a positive diagnosis were symptomatic. The cytological sampling was well tolerated by all patients. No complication was registered.

Conclusions: Liquid-based endometrial cytology can be considered an useful diagnostic method in the detection of endometrial pathology as a first-line approach, particularly if associated with transvaginal ultrasound.

Keywords: endometrial cytology, endometrial carcinoma, endometrial hyperplasia, histology, transvaginal ultrasound, liquid-based endometrial cytology

\section{Introduction}

Endometrial cancer is the fourth most common malignancy in women, and the most frequent gynae-

Correspondence:

F. Sesti, School of Medicine, Academic Department of Biomedicine \& Prevention and Clinical Department of

Surgery, Section of Gynecology, Tor Vergata University

Hospital, Viale Oxford 81, 00133 Rome, Italy

Tel./fax: +3906 20902 921;

E-mail:francesco.sesti@uniroma2.it cological cancer in developed countries. ${ }^{1,2}$ It frequently occurs in postmenopausal women, and abnormal uterine bleeding (AUB) is an early symptom. Only in the range between $0.07 \%$ and $0.6 \%$ are patients asymptomatic. $^{3-5}$ In about $10 \%$ of cases AUB is associated with endometrial cancer, but it can be caused by many other conditions, such as polyps, myomas, hormonal dysfunction and, most commonly in postmenopausal women, atrophy. ${ }^{6,7}$

Endometrial pathology can be identified using several methods, such as transvaginal ultrasound with 
or without Color-Doppler and 3-D analysis, hysteroscopy and endometrial biopsy, sonohysterography and the presence of endometrial cells in cervical cytology slides. ${ }^{8-17}$ Transvaginal ultrasound is the most common currently used first-line diagnostic method because it is well tolerated, but its accuracy is based on the endometrial thickness alone. ${ }^{1-13}$ Hysteroscopy with a biopsy is a second-line diagnostic tool, and is considered the gold standard in endometrial pathology detection. ${ }^{18-20}$

Traditionally, endometrial cytology was not considered sufficiently accurate in the detection of endometrial pathology, probably because of the common presence in the sample of confounding factors, such as blood and overlapping cells, which may hamper a correct diagnosis. ${ }^{21}$ Recently, endometrial cytology has been re-evaluated with the introduction of liquidbased techniques that reduce obscuring factors, increasing the diagnostic accuracy. ${ }^{22-24}$

In the literature, there is a need for additional trials to evaluate the diagnostic accuracy of liquid-based endometrial cytology (LBEC) in the evaluation of endometrial pathology. The present study aimed to compare LBEC with hysteroscopy and endometrial biopsy to assess its diagnostic accuracy in a series of postmenopausal women with AUB and asymptomatic postmenopausal women with thickened endometrium assessed by ultrasound as a screening test.

\section{Methods}

The trial was performed at general gynaecology clinics in the Department of Surgery, Tor Vergata University Hospital, Rome. From March 2007 to 2010, all postmenopausal women with AUB or asymptomatic women with thickened endometrium assessed by transvaginal ultrasound as a screening procedure were considered eligible for the study. Inclusion criteria were: (1) postmenopausal status; (2) the presence of AUB and/or thickened endometrium assessed by US (cut-off, at $4 \mathrm{~mm}$ without hormonal replacement therapy [HRT], at $5 \mathrm{~mm}$ with $\mathrm{HRT}$ or tamoxifen); and (3) a normal Pap smear. Exclusion criteria were: (1) previous endometrial pathology; (2) previous operative hysteroscopy; and (3) adnexal pathology at ultrasound.

Of 768 postmenopausal women referred to our gynaecology clinics, 121 fulfilled the inclusion criteria, and were recruited for the trial (Table 1). Twenty-one refused to participate. Written informed consent was obtained from each patient before entry in the study.

The study was approved by the local ethics committee. There was no financial interest or any arrangement with the companies producing the instruments used in the study or with competitor companies. There also was no direct payment to the authors from any source for the purpose of financing the writing of the manuscript, nor were there any other financial connections, direct or indirect, or other situations that might raise the question of bias in the work.

Cytological sampling was carried out by the same gynaecologist by brushing the uterine cavity using the Endoflower device (RIMOS; Mirandola, Modena, Italy) with no cervical dilation (Figure 1). The device measures $3 \mathrm{~mm}$ in diameter and consists of a mandrel with an umbrella-shaped tip. Some micro-holes are sited on the three curved thin arms. The mandrel, sliding inside an outer sheath, allows non-traumatic sampling of the entire uterine cavity, including the fundal and cornual regions. After having collected the endometrial cells, the tip is retracted inside the introducer to prevent cervical contamination and the device is removed. The umbrella-shaped tip is then immersed in the Cytolyt $^{\circledR}$ vial (formerly Cytyc Corporation [now Hologic ${ }^{\circledR}$ ] Boxborough, MA, USA), where it is shaken to allow the cells to release. The sample is centrifuged and the pellet containing the cells is transferred into a vial containing Preserv$\mathrm{Cyt}^{\circledR}$ (formerly Cytyc Corporation). With a succession of centrifugation and suspension to obtain mucolysis and hemolysis, blood and mucus are separated from the endometrial cells. At the end, the vial is processed using ThinPrep ${ }^{\circledR} 2000$ automated slide processor

Table 1. Distribution of patients according to inclusion criteria and age

\begin{tabular}{llll}
\hline Inclusion criteria & $\begin{array}{l}\text { Endometrial thickness }(\mathrm{mm}) \\
\text { mean } \pm \text { SD (range) }\end{array}$ & Number of patients & $\begin{array}{l}\text { Age (years) mean } \pm \text { SD } \\
\text { (range) }\end{array}$ \\
\hline Thickened endometrium & $8.08 \pm 3.85(5-23)$ & 70 & $62 \pm 7.27(47-81)$ \\
Thickened endometrium + AUB & $8.00 \pm 2.00(5-11)$ & 12 & $61 \pm 7.70(50-71)$ \\
AUB & $2.27 \pm 1.02(1-4)$ & 18 & $62 \pm 9.22(41-77)$ \\
\hline
\end{tabular}

AUB, abnormal uterine bleeding; SD, standard deviation. 


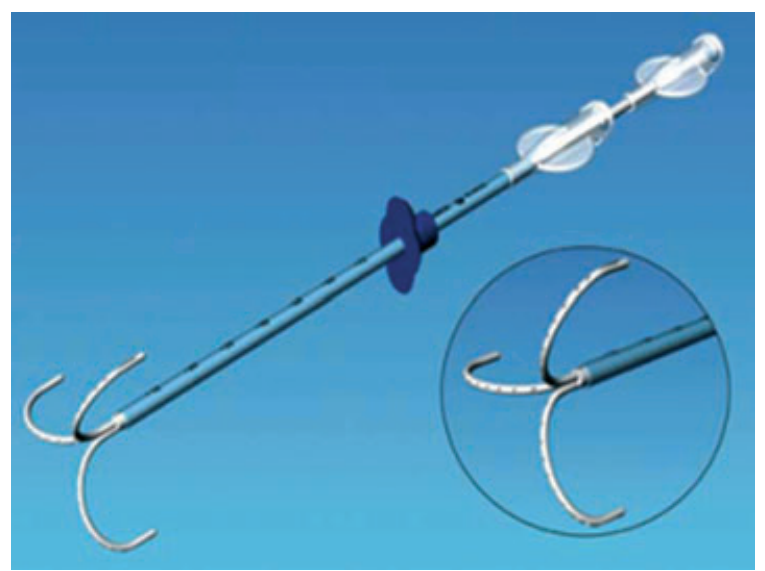

Figure 1. Endoflower sampling device.

(formerly Cytyc Corporation) and the slides stained with a Papanicolaou (Pap) method.

Office hysteroscopy was performed by the same gynaecologist using a 2.7-mm optic with saline solution distension after the cytological sampling. An endometrial biopsy was carried out using the Endoram device (RIMOS; Mirandola) measuring $3.8 \mathrm{~mm}$ in diameter. Endometrial samples were routinely fixed in neutral buffered formol, embedded in paraffin, and stained with haematoxylin and eosin.

The cytological and histological samplings were examined by the same pathologist. A numerical code was randomly assigned to each cytological and histological sample, and the pathologist was blinded to the correspondence between the code numbers and the cases examined. The assessments were made independently of each other, with the pathologist blinded to the result of the other assessment. The cytological criteria used in the cellular interpretation were according to existing techniques. ${ }^{22,25,26}$ The cytological findings were subdivided into four categories: negative, non-atypical hyperplasia, atypical hyperplasia and neoplasia (Figure 2a-d). Negative and non-atypical hyperplasia were considered as negative, and atypical hyperplasia and neoplasia as positive cytological results. The cytological findings were correlated with the histological results at hysteroscopy, which were considered as the gold standard; atypical hyperplasia or worse was taken as a positive outcome.

Statistical analysis was performed using the Statistical Program/SPSS for Windows, version 10 (SPSS Inc., Chicago, IL, USA). A double access table was created to evaluate the sensitivity of cytology (true positive/all positive biopsies), specificity (true negative/all negative biopsies), the positive predictive value (PPV) (true positive/all positive cytology results), the negative predictive value (NPV) (true negative/all negative cytology results) and diagnostic accuracy (true positive plus true negative/true and false positive plus true and false negative).

\section{Results}

The distribution of patients according to inclusion criteria is shown in Table 1 . The median age of women was $62 \pm 7.63$ years, median age at last menses $50 \pm 3.87$ years. Their median number of deliveries and miscarriages was $2 \pm 1.04$ and $0.71 \pm 1.18$, respectively. Some women had risk factors for endometrial cancer, such as diabetes, hypertension, smoking, HRT, Tamoxifen therapy and obesity (Table 2), whereas 57 did not have any risk (a)

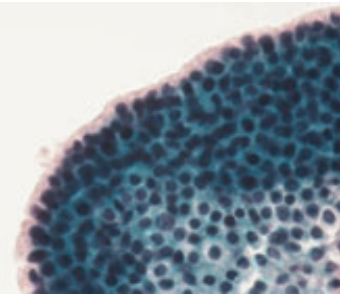

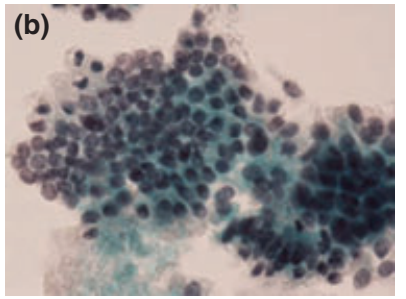
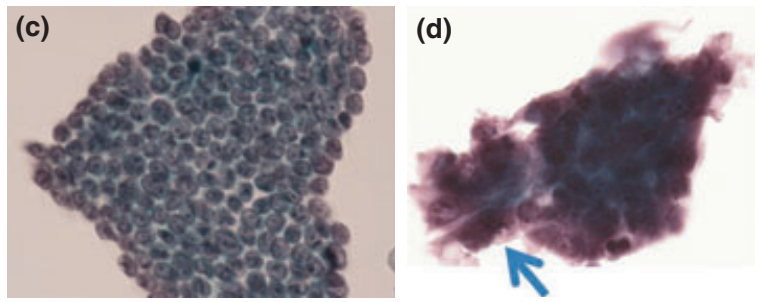

Figure 2. Liquid-based endometrial cytology (Papanicolaou $\times 60$ objective). (a) Normal endometrium: glandular cells with small, round, dense, pyknotic nuclei and scant cytoplasm. (b) Non-atypical endometrial hyperplasia: glandular cells characterized by apparent nuclear crowding, variation in the nuclear size with small nucleoli and evenly granular chromatin. (c) Atypical endometrial hyperplasia: glandular cells with enlarged round or ovoid nuclei, frequent nucleoli, dense irregular chromatin, nuclear overlapping and scant cytoplasm. (d) Endometrial carcinoma: sheets of glands with crowded cells, enlarged nuclei with frequent prominent nucleoli and an abnormal mitosis (arrow). 
Table 2. Risk factors of patients

\begin{tabular}{|c|c|c|c|c|c|}
\hline Risk factors & $\begin{array}{l}\text { Thickened } \\
\text { endometrium }\end{array}$ & $\begin{array}{l}\text { Thickened } \\
\text { endometrium + AUB }\end{array}$ & AUB & $\begin{array}{l}\text { Number } \\
\text { of patients }\end{array}$ & $\%$ \\
\hline Diabetes & 1 & 0 & 1 & 2 & 2 \\
\hline HRT & 2 & 0 & 0 & 2 & 2 \\
\hline Hypertension & 10 & 1 & 1 & 12 & 12 \\
\hline Smoking & 4 & 1 & 0 & 5 & 5 \\
\hline Tamoxifen & 9 & 0 & 1 & 10 & 10 \\
\hline Diabetes + hypertension & 0 & 0 & 2 & 2 & 2 \\
\hline Smoking + hypertension & 3 & 1 & 0 & 4 & 4 \\
\hline Tamoxifen + hypertension & 1 & 0 & 0 & 1 & 1 \\
\hline Tamoxifen + smoking & 2 & 0 & 0 & 2 & 2 \\
\hline Smoking + obesity & 0 & 1 & 0 & 1 & 1 \\
\hline Hypertension + obesity & 1 & 1 & 0 & 2 & 2 \\
\hline None & 37 & 7 & 13 & 57 & 57 \\
\hline Total & 70 & 12 & 18 & 100 & 100 \\
\hline
\end{tabular}

HRT, hormone replacement therapy; AUB, abnormal uterine bleeding.

Table 3. Correlation between histological and cytological results

\begin{tabular}{|c|c|c|c|c|}
\hline \multirow[b]{2}{*}{ Histology } & \multicolumn{4}{|l|}{ Cytology } \\
\hline & $\begin{array}{l}\text { Negative } \\
(n=36)\end{array}$ & $\begin{array}{l}\text { Non-atypical } \\
\text { hyperplasia }(n=47)\end{array}$ & $\begin{array}{l}\text { Atypical hyperplasia } \\
(n=10)\end{array}$ & $\begin{array}{l}\text { Neoplasia } \\
(n=5)\end{array}$ \\
\hline Negative or atrophy $(n=38)$ & 35 & & 3 & \\
\hline Non-atypical hyperplasia $(n=9)$ & & 9 & & \\
\hline Endometrial polyp $(n=38)$ & & 37 & & 1 \\
\hline $\begin{array}{l}\text { Submucosal leiomyoma }(n=1) \\
\text { Total negative or non-atypical hyperplasia }(n=86)\end{array}$ & & 1 & & \\
\hline Simple or complex atypical hyperplasia $(n=8)$ & 1 & & 7 & \\
\hline $\begin{array}{l}\text { Neoplasia }(n=4) \\
\text { Total atypical hyperplasia or neoplasia }(n=12)\end{array}$ & & & & 4 \\
\hline
\end{tabular}

factor. The latter were tested because they had AUB and/or thickened endometrium assessed by transvaginal ultrasound.

A correlation between cytological results and histological diagnosis is illustrated in Table 3. In 98 cases, endometrial cytology diagnosed five cases of endome-

Table 4. Diagnostic accuracy of the liquid-based endometrial cytology in the detection of atypical hyperplasia or worse as test and outcome positivity

\begin{tabular}{lll}
\hline Endometrial cytology & $\%$ & $95 \% \mathrm{CI}$ \\
\hline Sensitivity & 92 & $75-100$ \\
Specificity & 95 & $90-100$ \\
Diagnostic accuracy & 93 & $76-100$ \\
Positive predictive value & 73 & $67-80$ \\
Negative predictive value & 99 & $81-100$ \\
\hline
\end{tabular}

CI, confidence interval. trial cancer, 10 of atypical hyperplasia and 47 of nonatypical hyperplasia; 36 cases were negative. In two cases cytology was inadequate because of uterine cervical stenosis: office hysteroscopy with endometrial biopsy was performed, and histological diagnosis was atrophy in one patient, and an endometrial polyp in the other.

Histological diagnosis identified endometrial cancer in four patients, atypical hyperplasia in eight, endometrial polyps in 38, non-atypical hyperplasia in nine, submucousal myoma in one patient and no pathology in 38. In Table 4, the diagnostic accuracy of LBEC is reported. There was one false-negative and four falsepositive cytology results (three false positive if all degrees of abnormality are taken as positive). All the carcinomas had positive cytology. Among all the positive diagnoses (atypical hyperplasia or worse), only $42 \%$ of women were symptomatic. 


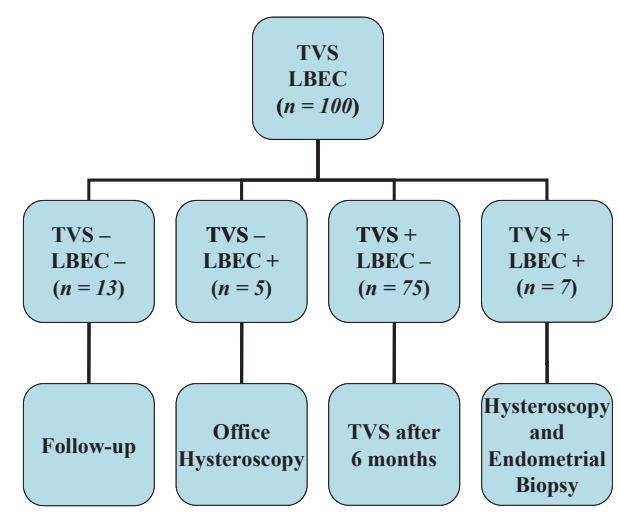

Figure 3. Diagnostic work up of postmenopausal women with $(n=30)$ and without $(n=70)$ abnormal uterine bleeding. TVS, transvaginal ultrasound; LBEC, liquid-based endometrial cytology.

The flow chart in Figure 3 demonstrates the clinical management based on the ultrasound and cytology results of women in this study.

\section{Discussion}

Endometrial cancer is the most frequent form of gynaecological neoplasia, but there is no reliable screening test for its early detection. Currently, transvaginal ultrasound is the first-line endometrial investigation, even although its diagnostic accuracy is only based on the endometrial thickness in relation to the cut-off considered.

With an endometrial thickness cut-off from 3 to $5 \mathrm{~mm}$, sensitivity goes from $90 \%$ to $100 \%$ and the NPV from $90 \%$ to $100 \%$, whereas specificity decreases from $78 \%$ to $60 \%$ and the PPV from $69 \%$ to $4 \% .^{11-13}$ When an endometrial thickening is detected by ultrasound, it is necessary to have access to office hysteroscopy with endometrial biopsy, which is considered the gold standard.

Traditional endometrial smears have not found a place in endometrial diagnostic workup because of their low diagnostic accuracy, with a sensitivity of about $78 \%$, specificity of $95 \%$, PPV of $56 \%$ and NPV of $98 \% .^{21}$ In spite of this, in Japan since 1987, conventional endometrial cytology has become a routine method for the initial examination of endometrial lesions. ${ }^{27}$

In order to improve the diagnostic accuracy of endometrial cytology, the thin layer method was proposed for the detection of endometrial disorders. In the first study published in 2003, Garcia et al. ${ }^{28}$ performed endometrial thin layer cytology in 203 symptomatic women reporting a specificity of $96 \%$ and sensitivity of $78 \%$ with an inadequate rate of $15 \%$. In the same year, Buccoliero et al. ${ }^{29}$ reported a cytohistological concordance of $98 \%$, and an inadequate rate of $18 \%$ in a population of 162 women before hysterectomy (55 symptomatic and 107 asymptomatic with prolapsed uterus). In 2007, the same authors performed liquid-based endometrial cytology in a population of 917, symptomatic and asymptomatic, pre- and postmenopausal women. They reported a sensitivity of $96 \%$, specificity of $98 \%$, PPV of $86 \%$ and NPV of $99 \%$. They emphasized that cytology provided sufficient material more frequently than a biopsy, and the difference was statistically significant. ${ }^{25}$ In the same year, they performed liquid-based endometrial cytology in 320 asymptomatic women reporting a sensitivity of $94 \%$, specificity of $95 \%$, PPV of $80 \%$ and NPV of $99 \%$. Cytological sample was adequate more often than endometrial biopsy. ${ }^{30}$

The findings of our study support those results, having obtained a sensitivity of $92 \%$, specificity of $95 \%$, PPV of $73 \%$ and NPV of $99 \%$. It is important to underline that all the cancers had positive cytology. The four false-positive results and the unique falsenegative finding were registered in the early stage of the study, when both the gynaecologist and the pathologist had to improve the technique of endometrial sampling and its cytopathological interpretation. The main reason probably consisted of the presence of cervical cells, a problem solved by cleaning the tip of the device with a gauze.

An additional improvement to LBEC in the evaluation of endometrial pathology could be obtained using immunocytochemical analysis with PTEN, $\beta$-catenin, and p53. ${ }^{31}$ Another possible application could result from the sampling obtained using a cytological device that provides cytology slides by means of membrane filtration and microbiopsies embedded in paraffin. Membrane-filtered cytology slides seem to be comparable to liquid-based cytology slides, but cheaper, and the sampler adequacy is still better than histology. ${ }^{32}$

On the basis of our results, and considering that only the $42 \%$ of women with a positive diagnosis (atypical hyperplasia or worse) were symptomatic, we believe that liquid-based endometrial cytology can be considered an useful diagnostic method in the detection of endometrial pathology as a first-line approach, particularly if associated with transvaginal ultrasound. Nevertheless, further studies are necessary to confirm its diagnostic accuracy as acceptable, and to 
investigate whether immunocytochemistry could significantly improve the diagnostic accuracy, so reducing the number of more expensive and invasive diagnostic tools.

If the use of diagnostic technique is to spread, it will probably be necessary to create a classification of the cytological results to improve the exchange of information between the clinician and pathologist. For this purpose, a Japanese group proposed a reporting format similar to the Bethesda System. ${ }^{27}$ They proposed considering the specimen adequacy as 'satisfactory for evaluation', 'less than optimal' and 'unsatisfactory for evaluation'. The results were categorized into four groups: '(1) negative; (2) atypical endometrial cells of undetermined significance (AECUS), suspicious for benign endometrial disease or simple endometrial hyperplasia (endometrial biopsy is not recommended); (3) atypical endometrial cells encompassing the spectrum of precursors to endometrial tumor (AEC-PEMT), suspicious for complex hyperplasia, simple or complex atypical hyperplasia, adenocarcinoma in situ (biopsy is recommended); and (4) positive: suspicious for a malignant tumor'. ${ }^{27}$ The value of this classification and the development of other classification systems should be subject of further clinical trials in order to identify an optimal categorization of endometrial pathology.

In conclusion, it is possible for liquid-based endometrial cytology together with transvaginal ultrasound to be an initial step in the diagnostic workup of AUB in postmenopausal women. If both methods are negative, women return to routine follow-up. If cytology alone is positive, women are offered office hysteroscopy. If ultrasound alone is positive, a repeat in 6 months is recommended. If both ultrasound and cytology are positive, women will undergo hysteroscopy with an endometrial biopsy.

\section{References}

1. Bray F, Loos AH, Oostindier M, Weiderpass E. Geographic and temporal variations in cancer of the corpus uteri: incidence and mortality in pre- and postmenopausal women in Europe. Int J Cancer 2005;117:123-31.

2. Kiviat NB, Wølner-Hanssen $\mathrm{P}$, Eschenbach DA et al. Endometrial histopatology in patients with cultureproved upper genital tract infection and laparoscopically diagnosed acute salpingitis. Am J Surg Pathol 1990;14: 167-75.

3. Feng L, Dingheng L. Evaluation of intrauterine disorders by hysteroscopy and transvaginal sonography. Gynaecol Endosc 2002;11:401-4.
4. Koss LG, Schreiber K, Oberlander SG et al. Screening of asymptomatic women for endometrial cancer. $C A$-Cancer J Clin 1981;31:300-17.

5. Hawwa ZM, Nahhas WA, Copenhaver EH. Postmenopausal bleeding. Lahey Clin Found Bull 1970;19:61-70.

6. Iatrakis G, Diakakis I, Kourounis G et al. Postmenopausal uterine bleeding. Clin Exp Obstet Gynecol 1997;24:157.

7. Nagele F, O'Connor H, Baskett TF et al. Hysteroscopy in women with abnormal uterine bleeding on hormone replacement therapy: a comparison with postmenopausal bleeding. Fertil Steril 1996;65:1145-50.

8. Vuento MH, Pirhonen JP, Mäkinen JI et al. Screening for endometrial cancer in asymptomatic postmenopausal women with conventional and colour Doppler sonography. Br J Obstet Gynaecol 1999;106:1229-30.

9. Odeh M, Vainerovsky I, Grinin V et al. Three-dimensional endometrial volume and 3-dimensional power Doppler analysis in predicting endometrial carcinoma and hyperplasia. Gynecol Oncol 2007;106:348-53.

10. Symonds I. Ultrasound, hysteroscopy and endometrial biopsy in the investigation of the endometrial cancer. Rev Gynecol Pract 2003;3:11-15.

11. Tabor A, Watt H, Wald N. Endometrial thickness as a test for endometrial cancer in women with postmenopausal vaginal bleeding. Obstet Gynecol 2002;99:663-70.

12. Gull B, Karlsson B, Milson I, Granberg S. Can ultrasound replace dilation and curettage? A longitudinal evaluation of postmenopausal bleeding and transvaginal sonographic measurement of the endometrium as predictors of endometrial cancer. Am J Obstet Gynecol 2003; 188:401-8.

13. Tsuda H, Kawabata M, Kawabata K, Yamamoto K, Umesaki N. Improvement of diagnostic accuracy of transvaginal ultrasound for identification of endometrial malignancies by using cutoff level of endometrial thickness based on length of time since menopause. Gynecol Oncol 1997;64:35-7.

14. Kufahl J, Pedersen I, Sindberg Eriksen P et al. Transvaginal ultrasound, endometrial cytology sampled by Gynoscan and histology of the uterine speciment. A prospective study in pre- and postmenopausal women undergoing elective hysterectomy. Acta Obstet Gynecol Scand 1997;76:790-6.

15. O' Connell LP, Fries MH, Zeringue E, Brehem W. Triage of abnormal postmenopausal bleeding: a comparison of endometrial biopsy and transvaginal sonohysterography versus fractional curettage with hysteroscopy. Am J Obstet Gynecol 1998;178:956-61.

16. Clark TJ, Mann CH, Shah N et al. Accuracy of outpatient endometrial biopsy in the diagnosis of endometrial cancer: a systematic quantitative review. BJOG 2002;109:313-21.

17. Wu HH, Schuetz MJ, Cramer H. Significance of benign endometrial cells in Pap smears from postmenopausal women. J Reprod Med 2001;46:795-8. 
18. Nagele F, O'Connor H, Davies A et al. 2500 outpatient hysteroscopies. Obstet Gynecol 1996;88:87-92.

19. Valli E, Zupi E, Marconi D et al. Outpatient diagnostic hysteroscopy. J Am Assoc Gynecol Laparosc 1998;5:397402.

20. Cicinelli E, Tinelli R, Colafiglio G et al. Reliability of the diagnostic fluid mini-hysteroscopy in the diagnosis of intrauterine pathologies. Minerva Ginecol 2009;61:4317.

21. Tsuda H, Kawabata M, Yamamoto K, Inoue T, Umesaki N. Prospective study to compare endometrial cytology and transvaginal ultrasonography for identification of endometrial malignancies. Gynecol Oncol 1997;65:383-6.

22. Buccoliero AM, Resta L, Napoli A, Taddei GL. Liquidbased endometrial cytology: the Florence and Bari experience. Pathologica 2009;101:80-4.

23. Friedlander MA, Rudomina D, Lin O. Effectiveness of the thin prep imaging system in the detection of adenocarcinoma of the gynecologic system. Cancer Cytopathol 2008;114:7-12.

24. Norimatsu Y, Kouda H, Kobayashi TK et al. Utility of thin-layer preparations in the endometrial cytology: evaluation of benign endometrial lesions. Ann Diagn Pathol 2008;12:103-11.

25. Buccoliero AM, Gheri CF, Castiglione FF et al. Liquidbased endometrial cytology: cyto-histological correlation in a population of 917 women. Cytopathology 2007; 18:241-9.

26. Norimatsu Y, Shimizu K, Kobayashi TK et al. Cellular features of endometrial hyperplasia and well differentiated adenocarcinoma using the Endocyte sampler: Diagnostic criteria based on the cytoarchitecture of tissue fragments. Cancer 2006;108:77-85.

27. Yanoh K, Norimatsu Y, Hirai Y et al. New diagnostic reporting format for endometrial cytology based on cytoarchitectural criteria. Cytopathology 2009;20:388-94.

28. Garcia F, Barker B, Davis J et al. Thin layer cytology and histopathology in the evaluation of abnormal uterine bleeding. J Reprod Med 2003;48:882-8.

29. Buccoliero AM, Caldarella A, Noci I et al. Thin layer method in endometrial cytology. Pathologica 2003;95:179-84.

30. Buccoliero AM, Castiglione F, Gheri CF et al. Liquidbased endometrial cytology: its possible value in postmenopausal asymptomatic women. Int J Gynecol Cancer 2007; 17:182-7.

31. Norimatsu Y, Miyamoto M, Kobayashi TTK et al. Diagnostic utility of phospatase and tensin homolog, $\beta$-catenin, and p53 for endometrial carcinoma by thinlayer endometrial preparations. Cancer 2008;114:155-64.

32. Manini C, Montironi PL, Magistris A, Stramignoni D. Diagnostic value of micro-hystology in endometrial brushing. Pathologica 2010;102:46-50. 